\title{
Melanomgefahr lässt sich bannen
}

Der Trend zu immer mehr MelanomPatienten hält weitweit an. Dass Präventionskampagnen bei Hautkrebs durchaus erfolgreich sein können, macht uns Australien vor.

W ie weltweit steigt auch in den meisten Ländern Europas die Melanom-Inzidenz weiter, wie aus der Auswertung der Daten durch die International Agency for Research on Cancer in Lyon hervorgeht. Betroffen sind vor allem die Länder Ost- und Südeuropas, aber auch in Slowenien, Dänemark und England stieg die Neuerkrankungsrate selbst bei jungen Menschen weiter. In Spanien zum Beispiel betrug die jährliche Zunahme der Inzidenz zwischen 1991 und 2000 bei Männern in der Altersgruppe der 25- bis 44-Jährigen 6,2 \% und bei den Frauen der gleichen Altersgruppe sogar 9,8\%. Auch in England liegen die jährlichen Zunahmen mit jeweils 5,8\% recht hoch.

Dagegen ist ein Trend zur Stabilisierung oder Verringerung der Inzidenzen in ei- nigen Hochrisikoländern wie in Neuseeland, Australien und Nordamerika sowie in Israel und Norwegen zu verzeichnen. Doch signifikant ist diese Entwicklung nach Angaben der Wissenschaftler nur in Australien in der Altersgruppe der 25- bis 44-Jährigen sowie in Island bei Frauen aller Altersgruppen. So nahm in Australien in den vergangenen zehn Jahren die Inzidenz bei Frauen um 1,8\% pro Jahr, bei den Männern um 1,2\% ab, in Island bei Frauen aller Altersgruppen um 4,3\%.

Zurückzuführen sind die positiven Trends wohl auf die Effekte von Präventionskampagnen. Bestes Beispiel ist Australien, wo bereits in den 1960er-Jahren TV-Spots gestartet wurden, in denen in einem Comic eine Möwe Zuschauer auffordert, Hemd, Hut und Sonnencreme beim Gang in die Sonne nicht zu vergessen. Diese Kampagne wurde inzwischen über das SunSmart-Hautkrebspräventionsprogramm um „Seek and Slide“ - also Schatten aufsuchen und Sonnenbrille aufsetzen! - erweitert und läuft noch heute.
Auch in Europa könnten die Menschen von Präventionskampagnen in den vergangenen Jahrzehnten profitieren, spekulieren die Wissenschaftler. Solche Kampagnen zielen vor allem auf Kinder, weil sie besonders gefährdet sind, später im Leben an einem Melanom zu erkranken. Allerdings sei der Zeitraum noch zu kurz, als dass sich Verhaltensänderungen aufgrund der Kampagnen auf die aktuellen Inzidenzzahlen niederschlagen könnten.

Fazit: Weltweit steigt die Melanom-Inzidenz seit mehreren Dekaden vor allem bei hellhäutigen Menschen an. Nur in einigen wenigen Ländern, allen voran in Australien, ist ein gegenläufiger Trend zu beobachten. Erstmals liegen jetzt Daten aus fast 40 Ländern auf vier Kontinenten vor, manche sogar über einen Zeitraum von mehr als 50 Jahren. Peter Leiner

Erdmann $\mathrm{F}$ et al. International trends in the incidence of malignant melanoma 19532008 - are recent generations at higher or lower risk? Int J Cancer. 2012 Apr 24. [Epub ahead of print]

\section{NSAR senken das Hautkrebsrisiko}

Patienten, die etwa wegen einer entzündlichen Gelenkerkrankung NSAR einnehmen müssen, profitieren eventuell noch in anderer Hinsicht davon: NSAR könnten - so eine aktuelle Studie aus Dänemark - vor Hautkrebs schützen.

S chon frühere Studien legten nahe, dass mit der Einnahme nicht-steroidaler Antiphlogistika speziell das Darmkrebsrisiko sinkt. Ob sich ein solcher Trend auch für andere Tumoren nachweisen lässt, untersuchten Epidemiologen von der Universität Aarhus für die drei Hautkrebsarten Basaliom, Spinaliom und malignes Melanom.

Sie analysierten die Verschreibungen von insgesamt 18.532 Patienten, die in den Jahren 1991 bis 2009 an Hautkrebs erkrankt waren, und verglichen sie mit denen von 178.655 Dänen einer Kontrollgruppe ohne Hautkrebs. Bei den diagnostizierten Hauttumoren handelte es sich um 1.974 Spinaliome, 13.316 Basaliome und 3.242 Fällen maligne Melanome.

Wem dreimal oder öfter nicht-steroidale Antiphlogistika vom Arzt verschrieben worden waren, der hatte ein $15 \%$ geringeres Risiko für ein Spinaliom und ein $13 \%$ geringeres Risiko für ein Melanom im Vergleich zu Personen mit zwei oder weniger Verschreibungen. Beim Basalkarzinom hingegen brachte die NSAREinnahme keinen generellen Vorteil. Lediglich für Basaliome an nicht-sonnen-

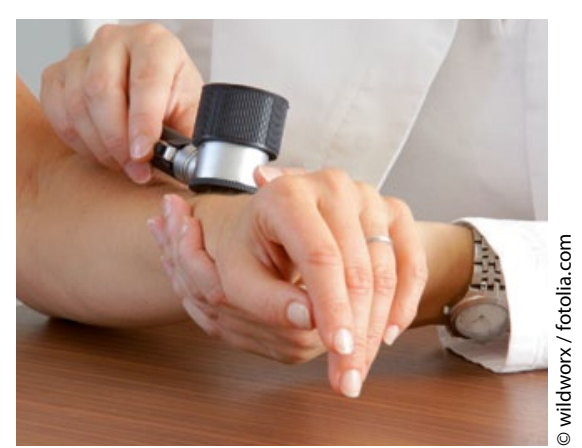

Bei Patienten, die NSRA einnehmen, wird seltener Hautkrebs diagnostiziert. exponierten Hautarealen sank das Risiko unter der Einnahme um 15-21\%.

Das Risiko verringerte sich umso mehr, je länger und je höher dosiert NSAR eingenommen worden waren, weshalb die Autoren einen kumulativen biologischen Effekt vermuten. Wirkstoff-spezifische Unterschiede hinsichtlich des protektiven Effekts ließen sich beim Spinaliom nicht und beim malignen Melanom nur für die neueren COX-2-Hemmer erkennen, bei denen ein solcher ausblieb.

Fazit: Unter der Einnahme von NSAR sank das Risiko, an Spinaliomen oder malignen Melanomen zu erkranken, in Abhängigkeit von Dosis und Dauer der Einnahme. Auch wenn diese Ergebnisse mit Vorsicht zu werten sind, hoffen die Autoren, dass nun auch andere Forscher die NSAR auf einen potenziell krebsprotektiven Effekt hin überprüfen.Dagmar Kraus

Johannesdottir SA et al. Nonsteroidal anti-inflammatory drugs and the risk of skin cancer. A population-based case-control study. Cancer. 2012 May 29 [Epub ahead of print]. 